\title{
Corneal ulcers associated with FHV-1 in cats
}

\author{
Sergey Kartashov ${ }^{1, *}$, Evgeniya Kartashova $^{2}$, Alexander Butenkov ${ }^{1,2}$, Anastasia \\ Rakityanskaya ${ }^{1}$, Marina Petrova ${ }^{1}$, Maria Oboeva $^{1}$, and Maria Sultanova ${ }^{1}$ \\ ${ }^{1}$ Federal State Budget Educational Institution of Higher Education "Don State Technical University", \\ 344000, Rostov-on-Don, Russian Federation \\ ${ }^{2}$ Limiter Liability Company "VITAVET”, Russian Federation
}

\begin{abstract}
Corneal ulcer is a common pathology of the anterior part of the eye in cats, of various etiologies, but clinically manifested stereotypically, by the loss of corneal epithelium with a positive fluorescein test and pain. An analysis of 184 cases of various problems of the organs of vision revealed that the incidence of this pathology in the Rostov region is more than $70 \%$. Corneal ulcers of any etiology with simultaneous infection caused by FHV-1 respond better to therapy with systemic antiviral and anti-collagenase drugs. Combined therapy of corneal ulcers in cats with the simultaneous administration of "Famciclovir" and heterogeneous serum significantly reduces the recovery time of the corneal epithelium.
\end{abstract}

\section{Introduction}

Corneal ulcers in cats are urgent conditions that require quick diagnosis and effective therapy. An undetected corneal ulcer can rapidly progress, deepen with the threat of perforation, the development of uveitis and, as a result, loss of eye function [1,2].

Regardless of the nature of the ulcer, when it is detected by a fluorescein test, a full ophthalmological examination should be performed to make sure that the correct diagnosis is established and that any concomitant eye disease is recognized and treated properly [3, 4, 5]. In the presence of an ulcer on the cornea, if the eyelids and intraocular structures have also not been thoroughly examined, it is possible to incorrectly diagnose the root cause of the ulcer on the cornea (trichiasis, uveitis) [4]. If the anomaly of the eyelids is not eliminated, the corneal ulcer will not heal and will progress to perforation. In addition, if the intraocular structures have not been thoroughly investigated, reflex uveitis resulting from ulceration of the cornea may not be diagnosed, remain uncontrolled, and cause more serious complications that threaten vision, such as synechia, cataracts, or glaucoma. A thorough study of all the external and internal structures of both eyes should be carried out, even if clinical manifestations have a one-sided problem $[4,5,6]$.

In veterinary ophthalmology, a corneal ulcer is called the destruction of the corneal epithelium with the loss of corneal stroma at different depths. In sick cats, acute unilateral blepharospasm and epiphora are often observed. Due to reflex uveitis, irritation of the corneal endings of the trigeminal nerve with myosis and the development of anisocoria is possible $[1,6,7,8]$. Depending on the severity and duration of the disease, a slit lamp may

* Corresponding author: vera.murgul@mail.ru 
show turbidity of the liquid in the anterior chamber of the eye of varying severity, which characterizes the anterior uveitis. If corneal perforation has occurred, fluid leak from the eye, a sharp decrease in intraocular pressure, hyphema, or loss of the iris, are possible. When examining in a dark room using bright light and magnification, corneal irregularities can be easily manifested, which is even better detected in the rays of a slit lamp, uneven reflection of light from the cornea is clearly visible (Purkinje image) [9, 10], which indicates a corneal surface pathology. Corneal edema of varying severity is a common clinical symptom in corneal ulcers, but the fluorescin test is a classic method for detecting corneal ulcers and their depth. Fluorescin stains well the structures of the corneal stroma unprotected by the epithelium, this is the main diagnostic tool for determining the depth of the ulcer $[9,11]$. Ultrasound examinations of the eye become indispensable; in case of the anterior segment damage of the eye and opacity of the cornea and fluid of anterior chamber of the eye, for example, severe corneal edema or hyphema prevents adequate intraocular examination by optical methods [1].

When revealing a corneal ulcer, the next step is its clinical characteristic: size, shape, depth and breadth of the corneal ulcer; it is necessary to find out what is the main cause of the ulcer; the condition of the tissues surrounding the cornea (signs of inflammation); the proximity of the ulcer to the limb (the possibility of rapid vascularization of the affected area, which has a beneficial effect on the course of the disease) [10].

Diagnosis is aimed at identifying the causes of the ulcer, and therapy is aimed at eliminating the etiological factor. With superficial, uncomplicated corneal ulcers, the prevention of corneal infections and the treatment of reflex uveitis with the administration of topical antibiotic therapy with a wide spectrum of action 4-6 times a day, mydriatic cycloplegia with atropine are carried out. Systemic analgesics can significantly alleviate pain and relieve pain phenomena (blepharospasm, epiphora) [14]. Anesthetics are used only for diagnostic purposes because their prolonged use negatively affects the healing of corneal ulcers. Indications for surgical repair of corneal ulcer are: loss of $50 \%$ or more of corneal stroma; rapid progression of ulcer; an infected ulcer (as evidenced by yellow to white cellular corneal infiltration, significant corneal edema, mucopurulent discharge from the eyes, and moderate to severe uveitis); descemetocele; perforation of the cornea. Various methods of surgical recovery include conjunctival transplants, corneal scleral transposition, and keratoplasty $[4,12,14]$.

For complex corneal ulcers, local and systemic antibiotic therapy should be based on the results of bacteriological studies and the sensitivity of the microflora obtained from the ulcer bed to the antibiotic. Local antimicrobials can be administered hourly for infected or rapidly progressing ulcers in the initial stages of treatment. Atropine is administered 2 to 4 times a day until pupillary dilatation is achieved, and then only as needed. Antiprotease drugs ( $\mathrm{N}$-acetylcysteine, fresh serum, EDTA) remain an important therapeutic component in the treatment of corneal ulcers, they are used locally every 2-6 hours to inhibit the progression of corneal stroma destruction by bacterial proteases. Systemic antibiotic therapy is useful if conjunctival transplantation has been performed or corneal perforation has occurred. Systemic non-steroidal anti-inflammatory drugs (NSAIDs) control uveitis and relieve soreness from inflamed tissues, which has a beneficial effect on the course of the disease. However, caution should be exercised when administering systemic NSAIDs to cats due to possible red bone marrow depression, ulceration in the gastrointestinal tract, followed by bleeding, vomiting, and diarrhea. Local and systemic corticosteroids are contraindicated in complex or infected corneal ulcers, since they delay wound healing and increase collagenase activity [15].

In a domestic cat population, FHV-1 infection is widespread in most countries of the world, and is the most common cause of eye disease complications. Many authors believe that any corneal ulceration, inflammation of the uveal tract in a cat is secondary to FHV-1 
infection. Based on the clinical factors and the results of the polymerase chain reaction (PCR), it was found that primary infection usually occurs in kittens, and in $80 \%$ of cases it progresses to latent infection. In the future, a latent infection is periodically reactivated, clinically manifested including keratitis. In $45 \%$ of spontaneous reactivation of FHV-1 infection, the pathogen can be transmitted between cats in close contact, through body fluids, in particular, aerogenic way. Cats with acute FHV-1 infection secrete the largest number of viral particles; however, it has been proven that latently infected cats can also secrete the virus and infect susceptible animals [1, 11]. Infection (FHV-1) is widespread among domestic cat populations and is believed to be the most common cause of cat eye disease complications, and some have suggested that any corneal ulceration, inflammation of the uveal tract in a cat is secondary to FHV-1 infection [1].

Despite recent advances in understanding the pathogenesis of corneal ulcers in cats, developed various treatment regimens, corneal ulcers in these animals are often complicated by descemetocele, perforation and blindness, which determines the relevance of our study.

\section{The purpose and objectives of the study}

To identify the occurrence frequency of corneal ulcers in cats in the Rostov Region, to analyze the etiology of this disease, to find out the effectiveness of various approaches in the treatment of corneal ulcers associated with FHV-1.

\section{Material and research methodology}

We conducted an analysis of 184 case histories of cats with an ophthalmological problem, which applied for treatment to the veterinary clinic of Vita in Rostov-on-Don and Taganrog, from 01.08.18 to 02.05.19. For differential diagnosis and clarification of the disease etiology, all animals underwent a full clinical survey and a complete ophthalmological examination:

- research on rhinotracheitis (FHV-1), calicivirus (FCV), mycoplasmosis and chlamydia of cats was performed by PCR in an external laboratory.

- The animals underwent a complete ophthalmological examination, which included: eye examination, while paying attention to the closure completeness of the palpebral fissure, the presence of exophthalmos or enophthalmos, the position and presence of lacrimal openings, the condition of the lacrimal sac area, the cranial reflexes of the $\mathrm{V}$ pair of cranial nerves (touching the cornea), VII pair of cranial nerves (patting on the temple) were determined; tonometry was performed with an ophthalmic tonometer "TonoVet".

The following tests were carried out for all animals:

- Schirmer I test,

- Norn lacrimal film rupture test,

- Jones 1 test,

- fluorescin test, if a fluorescin test was negative, than a lissamine test was performed;

- examination of the eye structures was carried out using an ophthalmic magnifier, magnification $\times 6$, when examining the skin of the eyelids, attention was paid to the condition of the eyelid edge, the presence of dystrichia, ectopic eyelashes, the state of the ducts of the meibomian glands and the nature of the discharge from them; when examining the conjunctiva, they paid attention to the discharge from the eyes, conjunctival edema, type of conjunctival hyperemia, type of scleral hyperemia, if necessary, a test was performed with irifrin, the presence of trichiasis of the lacrimal meat region was noted; 
- examination of the cornea was performed using a Shin Nippon XL-1 slit lamp, the state of the corneal surface, its transparency, the presence of defects, thickenings, overlays were noted, the thickness of the precorneal lacrimal film was determined;

- examination of the iris was performed with a Shin Nippon XL-1 slit lamp for the presence of synechia, an inversion of the pigment leaf of the iris, the state of the vascular apparatus of the iris were noted;

- examination of the anterior chamber of the eye was performed with a Shin Nippon XL-1 slit lamp; the state of fluid in the anterior chamber of the eye, the depth of the anterior chamber, the presence of foreign objects, pus, blood in the anterior chamber were determined;

- examination of the pupil and pupil-motor reactions were determined for white, blue and red colors, the rate of reaction of the pupil to mydriatics was noted;

- examination of the lens was performed with a Shin Nippon XL-1 slit lamp;

- fundus examination (ophthalmoscopy) was performed with a Smartscope m-5 fundus camera for the presence of hyper- and hyporeflective zones, the presence of hemorrhages, exfoliation, retinal edema, signs of its dysplasia; the state of the fundus vessels, its tortuosity, perivascular edema, hemorrhages and proliferative processes were noted;

- examination of the optic nerve disc was performed with a Smartscope m-5 fundus camera;

- ultrasound was performed on a DC-N6 ultrasound scanner using a linear sensor with a frequency of 10-14 MHz, the anteroposterior size of the eyeball, the thickness of the cornea were determined: the integrity of the cornea, inclusions or foreign objects in the cornea, the anteroposterior size of the anterior chamber of the eye, and the contents of the anterior chamber of the eye, the condition of the iris and the region of the corneoscleral junction, the condition of the ciliary body, topography, shape, transparency and anteroposterior size of the lens, damage to the lens capsule were noted.

\section{Results and discussion}

An analysis of 19378 case histories of animals that had been treated at the "VITA" Veterinary Clinic in Rostov-on-Don and Taganrog was carried out. It was found that 443 animals with pathology of the organs of vision were identified, of which 248 dogs and 184 cats with ophthalmic pathology at the age of 1 month to 15 years. Analysis of ophthalmic morbidity among cats showed that for six months, 129 cats were diagnosed with corneal ulcer. The following subgroups were selected from this group: indolent ulcers - 3; deep corneal ulcers - 51; superficial ulcers - 69; corneal ulcers with descemetocele - 6 .

We conducted an analysis of the occurrence frequency of this pathology among certain cat breeds, as well as sexual predisposition (Table 1).

Table 1. Frequency of occurrence depending on the breed, age and sex of cats.

\begin{tabular}{|c|c|c|}
\hline Character & number of animals & $\begin{array}{c}\text { percentage of occurrence } \\
(\%)\end{array}$ \\
\hline \multicolumn{3}{|c|}{ Breed } \\
\hline Siberian breed & 4 & $3.1 \%$ \\
\hline Siamese breed & 5 & $3.9 \%$ \\
\hline Cornish Rex & 3 & $2.3 \%$ \\
\hline mestizo & 45 & $34.9 \%$ \\
\hline Don Sphinx & 6 & $4.6 \%$ \\
\hline British breed & 21 & $16.3 \%$ \\
\hline Scottish fold & 8 & $6.3 \%$ \\
\hline Scottish breed & 18 & $13.9 \%$ \\
\hline
\end{tabular}




\begin{tabular}{|c|c|c|}
\hline Maine Coon & 9 & $7 \%$ \\
\hline Persian breed & 3 & $2.3 \%$ \\
\hline Canadian Sphinx & 7 & $5.4 \%$ \\
\hline \multicolumn{3}{|c|}{ Age } \\
\hline less than a year & 25 & $19.4 \%$ \\
\hline 1-5 years old & 58 & $45 \%$ \\
\hline 5-10 years old & 32 & $24.8 \%$ \\
\hline more than 10 years & 14 & $10.8 \%$ \\
\hline \multicolumn{2}{|c|}{ Sex } \\
\hline females & 45 & $34.9 \%$ \\
\hline males & 84 & $65.1 \%$ \\
\hline
\end{tabular}

Among the breeds, this pathology was most prevalent in British and Scottish cats, and the greater occurrence frequency of this pathology in mestizos, rather indicates their greater prevalence in our region than thoroughbred cats.

The distribution estimate of corneal ulcers between intact and non-neutered cats was also carried out (Figure 1). According to our data, corneal ulcers in non-neutered cats are twice as common as in neutered cats, which may be explained by the more aggressive behavior of cats, which are regular participants in fights for identifying a dominant male worthy of a female. Most often, a corneal ulcer was found in cats aged 1 to 5 years, most likely due to the most physically active period in the life of these animals.

\section{Assessment of the repoductive function of cats}

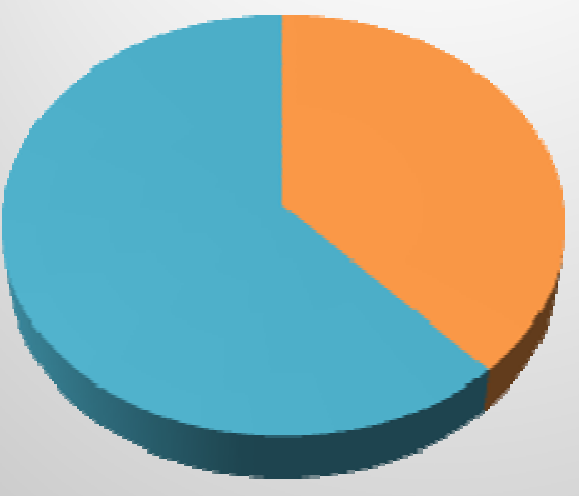

Sterilized cats

- Unsterilized cats

Fig. 1. Incidence of corneal ulcers in sterilized and unsterilized cats.

We performed an analysis of the epithelialization period duration of extensive corneal ulcers in cats, with PCR confirmed diagnosis of herpes virus infection. Material was taken into sterile containers from the surface of the ulcers. One group of cats received the therapy prescribed in the ophthalmology room of the veterinary clinic of "Vita", Rostov-on-Don, and included systemic antiviral therapy: "Famvir" (famciclovir) $50 \mathrm{mg} / \mathrm{kg}$ once a day; anticollagenase preparations - heterogeneous serum (dog serum) for instillation in both eyes 56 times a day; local antibiotics - "Phloxal" (ofloxacin 0.3\%), "Fucitalmic" (fusidic acid $1 \%$ ) three times a day; systemic non-steroidal anti-inflammatory drugs - "Rimadil" (caprofen 5\%); root protectors - "Korneregel" (dexpanthenol 5\%), "Balarpan" (glycosaminoglycans $0.01 \%$ ). 
The second group of animals with a similar confirmed diagnosis was treated in the Vita Taganrog clinic according to the following scheme: systemic antibiotic therapy - "Unidox solutab" (doxycycline) $10 \mathrm{mg} / \mathrm{kg}$; local antibiotics - "Phloxal" (ofloxacin 0.3\%), "Fucitalmic" (fusidic acid 1\%) three times a day; systemic non-steroidal anti-inflammatory drugs - "Rimadil" (caprofen 5\%); root protectors - "Korneregel" (dexpanthenol 5\%), "Balarpan" (glycosaminoglycans 0.01\%).

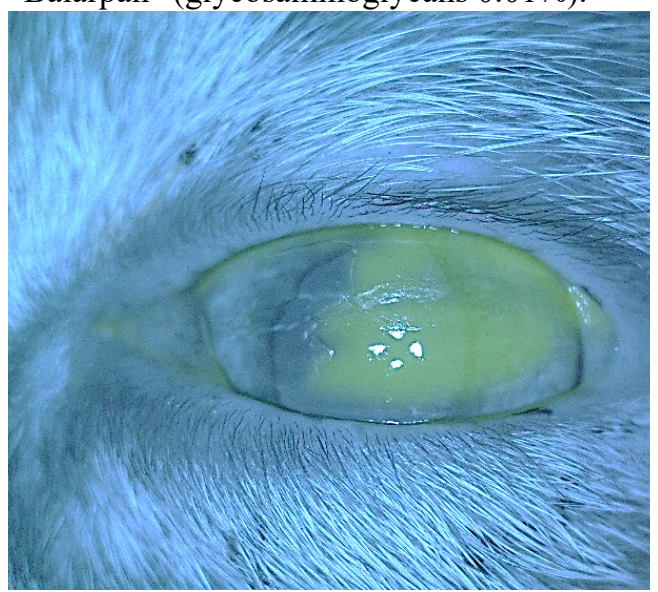

Fig. 2. Deep corneal ulcer associated with FHV1 , positive fluorescin test.

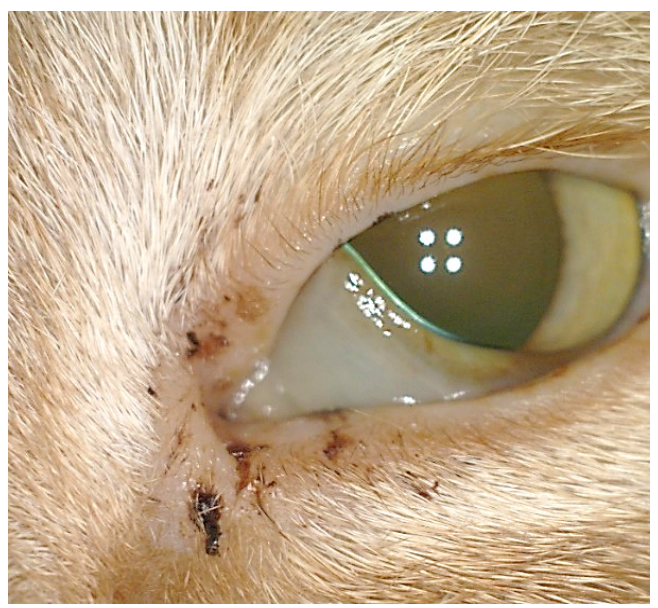

Fig. 4. The same animal as in Figure 2 after therapy, the disappearance of clinical signs.

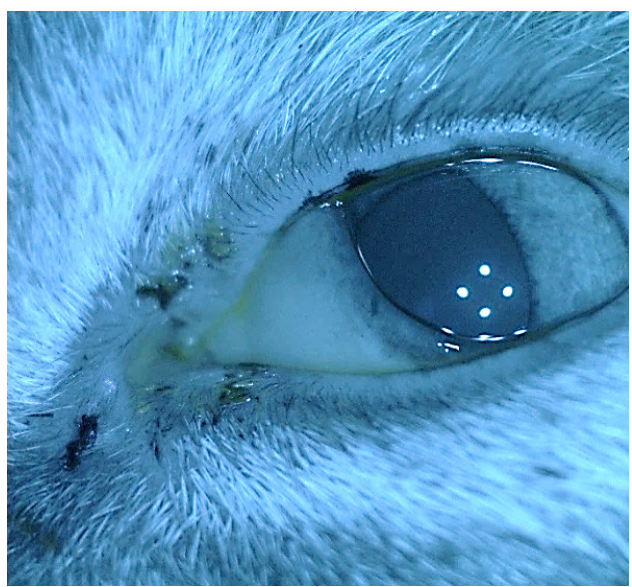

Fig. 3. The same animal as in Figure 2 after therapy, negative fluorescin test.

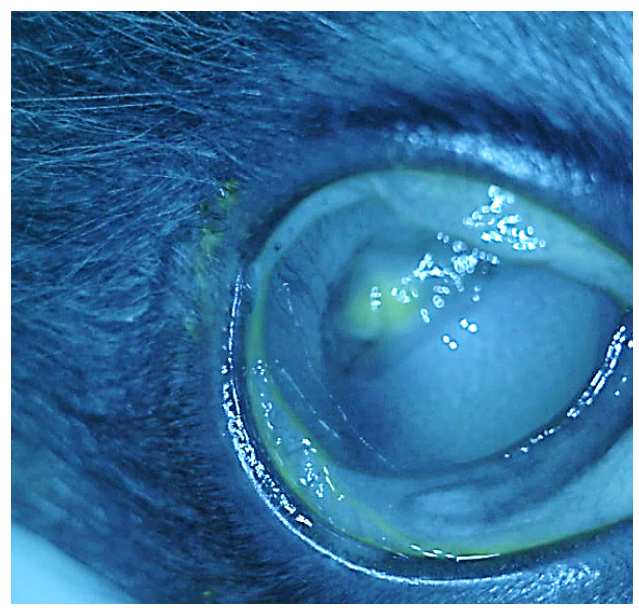

Fig. 5. Deep corneal ulcer, corneal edema, uveitis associated with FHV-1, positive fluorescin test. 


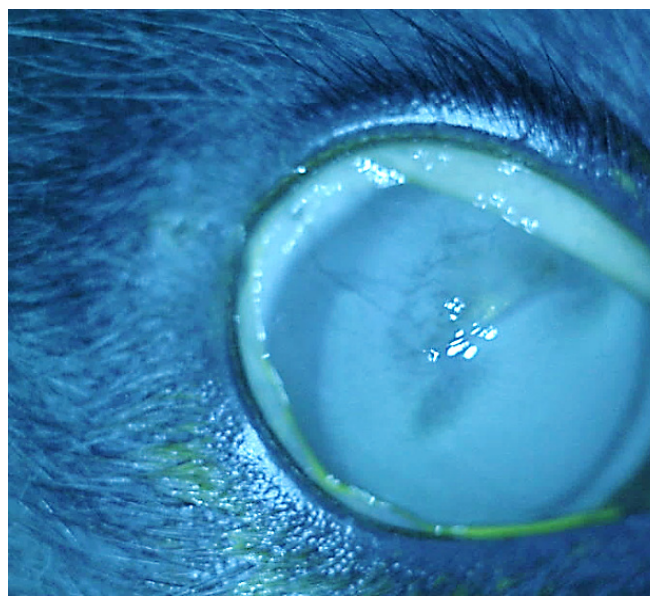

Fig. 6. The same animal as in Figure 5 after therapy, negative fluorescin test, corneal vascularization.

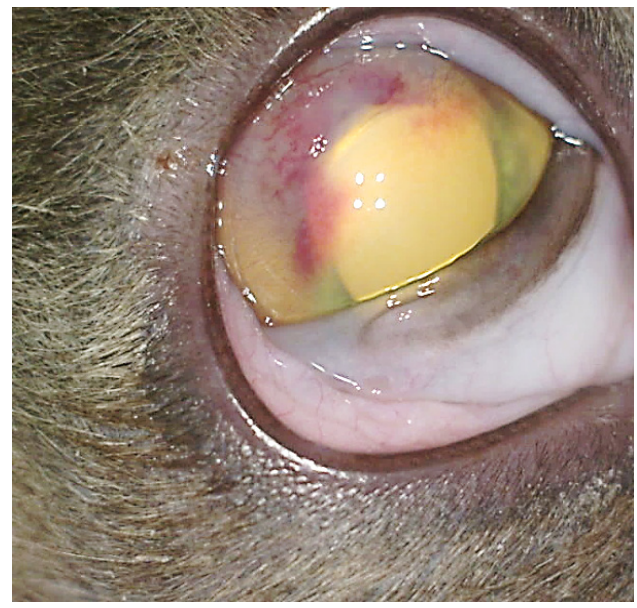

Fig. 7. The same animal as in Figure 5 after therapy, vascularization and clouding of the cornea.

In the "Vita" clinic in Rostov-on-Don, 11 FHV-1 positive cats were detected. Corneal ulcers associated with FHV-1, as a rule, have a pronounced clinical manifestation (blepharospasm, epiphora), with the formation of extensive defects in the corneal epithelium (Figure 2). The treatment regimen for these cats included a systemic antiviral drug (famciclovir) at a dose of $50 \mathrm{mg} / \mathrm{kg}$ once a day, local antibiotic therapy on the background of heterogeneous (dog) serum with a frequency of use of 5-6 times a day. The period of ulcer epithelization ranged from 5 to 9 days. Against the background of the therapy, positive dynamics was noticeable for five days. The clinical signs of the disease disappeared, and the fluorescin test became negative (Figure 3.4).

In the second group there were $16 \mathrm{FHV}-1$ positive cats. These cats underwent systemic and local antibiotic therapy, and corneoprotectors were prescribed. Tracking the dynamics of this pathology, a slow healing of corneal defects was revealed. The duration of therapy ranged from 10 to 14 days (Figure 5-7).

During the two-week therapy, incomplete epithelization of the corneal defect was observed. Fluorescin test remained positive for up to 10 days. It can be noted that with systemic antiviral therapy, as well as local use of anticollagenase drugs, a more rapid effect in the treatment of this pathology was observed.

\section{Conclusion and inference}

After analyzing 184 case histories of cats with an ophthalmological problem, it was found that the incidence of corneal ulcers was higher in cats of the British and Scottish breeds than in other breeds that were applied to a veterinary ophthalmologist. It was found that in intact cats, a corneal defect is more common than in castrated cats, which is characteristic of confrontational behavior. It was also established that frequent patients of the veterinary ophthalmologist were cats aged 1-5 years.

Ulcerative lesions in cats in $21 \%$ of cases are associated with the presence of FHV-1 infection, which, when reactivated, is manifested by damage to the cornea, most often with the destruction of its epithelium. In the treatment of such cats, it is recommended to use first of all antiviral therapy, supplementing it with symptomatic treatment: moisturizing the cornea, anti-inflammatory drugs and antibiotics. In our work for this purpose we used 
"Famciclovir". Combined therapy with "Famciclovir" and serum significantly reduced the recovery time of the corneal epithelium, according to our data, up to 5 days.

\section{References}

1. A. Groth, M. Contreras, H. Kado-Fong, Vet Ophthal, 17, pp. 268-274 (2014)

2. F. Goulle, J. Sm Anim Pract, 53, pp. 34-43 (2012)

3. E. Scott, R. Carter, J. Am Anim Hosp Assoc., 50, pp. 112-118(2014)

4. E. Bentley, C. Murphy, Vet Clin North Am Sm Anim Pract., 34, pp. 623-638 (2014)

5. National Research Council (U.S.), Guide for the care and use of laboratory animals. Washington, D.C.: National Academies Press, 8-th Ed. (2011)

6. O. Balland, A. Poinsard, F. Famose, Vet. Ophthalmol, 19, pp. 454-463(2016)

7. D. Chow, H. Westermeyer, Vet. Ophthalmol, 19, pp. 357-366 (2016)

8. P. Steagall, B. Monteiro-Steagall, P.Taylor, J. Vet. Interi n Med 28, pp. 762-770 (2014)

9. M. Lessels, Journal of Feline Medicine and Surgery, 11 (1), pp. $40-48$ (2009)

10. D. Maggs, Vet Clin North Am Small Anim Pract, 40 (6), pp. 1055-62(2010)

11. S. Thomasy, C. Lim, C. Reilly, P. Kass, M. Lappin, D. Maggs, Am J. Vet Res., 72 (1) pp. 85-95 (2011) 